\title{
NGC 2359: VLA H I and radio continuum observations
}

\author{
Cristina Cappa ${ }^{1,2,4}$, W. Miller Goss ${ }^{3}$, Virpi S. Niemela ${ }^{2,5,6}$, \\ and Pablo G. Ostrov 2,5 \\ ${ }^{1}$ Instituto Argentino de Radioastronomía, \\ C.C. 5, 1894 Villa Elisa, Argentina \\ ${ }^{2}$ Facultad de Ciencias Astronómicas y Geofísicas, \\ U.N. La Plata, Paseo del bosque s/n, 1900 La Plata, Argentina \\ ${ }^{3}$ National Radio Astronomy Observatory, \\ PO Box 0, Socorro, NM 87801, USA
}

\section{Introduction}

NGC 2359, a H II region located at a distance of $5 \mathrm{kpc}$ from the Sun (e.g., Goudis et al. 1994), appears as a ring like nebula surrounding HD 56925, a Wolf-Rayet star of type WN4. The nebula consists of a filamentary shell, a southern bar and streamers of diffuse gas ( $c f$. Schneps et al. 1981, SHWB). Here we report the results of $\mathrm{H}_{\mathrm{I}} 21-\mathrm{cm}$ line and radio continuum observations in the direction of NGC 2359.

\section{Observations}

Our observations were performed with the $N R A O^{7}-V L A$ synthesis telescope. The radio continuum image, obtained at $1465 \mathrm{MHz}$ using the $\mathrm{C}$-and D-arrays, has an angular resolution of $\sim 30^{\prime \prime}$ and covers a region of $30^{\prime \prime}$. The $\mathrm{HI} 21-\mathrm{cm}$ line data, obtained with the D-configuration, cover the same region with angular and velocity resolutions of $\sim 45^{\prime \prime}$ and $1.3 \mathrm{~km} \mathrm{~s}^{-1}$. In addition, optical CCD images through narrow band filters centered at the nebular emission lines of $\mathrm{H} \alpha$, [OIII] and [SII], were obtained with the Curtis-Schmidt Telescope at CTIO, Chile. These images are $\sim 30^{\prime}$ square.

\section{Results}

The radio continuum image of NGC 2359 shows an excellent correspondence with the optical features. Table 1 lists the emission measure $E M$, the electron density $n_{e}$ and the ionized mass $M_{i}$ together with the volume filling factor $f$ derived for the filamentary shell, the southern bar, the streamers, and the weak radio continuum emission region (at a level of $1 \mathrm{mJy} \mathrm{beam}^{-1}$ ) that surrounds the ring nebula. The amount of ionized gas in the filamentary shell indicates that it

\footnotetext{
${ }^{4}$ Member of Carrera del Investigador, CONICET, Argentina

${ }^{5}$ Visiting Astronomer, CTIO, NOAO, operated by AURA, Inc., under agreement with NSF

${ }^{6}$ Member of Carrera del Investigador, CIC, Prov. Buenos Aires, Argentina

${ }^{7}$ NRAO is a facility of NSF operated under agreement by AUI
} 
Table 1. Physical parameters of ionized gas in NGC 2359

\begin{tabular}{lccccc}
\hline component & $\begin{array}{c}S \\
(\mathrm{Jy})\end{array}$ & $\begin{array}{c}E M \\
\left(10^{3} \mathrm{pc} \mathrm{cm}^{-6}\right)\end{array}$ & $f$ & $\begin{array}{c}n_{e} \\
\left(\mathrm{~cm}^{-3}\right)\end{array}$ & $\begin{array}{c}M_{i} \\
\left(\mathrm{M}_{\odot}\right)\end{array}$ \\
\hline shell & 0.85 & 3.8 & 0.03 & 120 & 70 \\
southern bar & 0.58 & 9.0 & $0.3-0.5$ & 60 & $95-120$ \\
streamers & 0.78 & 3.6 & 1 & $10-50$ & $105-280$ \\
seak region & 0.38 & 0.3 & 1 & $\sim 3$ & $\sim 600$ \\
\hline
\end{tabular}

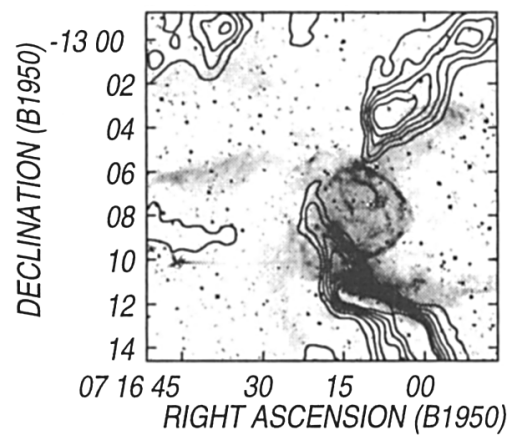

Figure 1. Contours of the $\mathrm{H} \mathrm{I} 21-\mathrm{cm}$ line emission distribution at $54 \mathrm{~km} \mathrm{~s}^{-1}$ superposed to the grey-scale optical $\mathrm{H} \alpha$ image of NGC 2359. The contour lines correspond to $5,15,25,35,45$ and $55 \mathrm{mJy} \mathrm{beam}^{-1}$ or $1.3,3.9,6.5,9.1,11.7$ and $14.3 \mathrm{~K}$. The synthesized beam is $57^{\prime \prime} \cdot 7 \times 40^{\prime \prime} 6$.

mostly consists of swept-up interstellar gas. The excitation parameter indicates that HD 56925 alone suffices to ionize the gas.

Analysis of the H I 21-cm line images reveals features connected with the ring nebula within the (LSR) velocity range from 46 to $67 \mathrm{~km} \mathrm{~s}^{-1}$. Two $\mathrm{H} \mathrm{I}$ structures appear most clearly related to the nebula. One of them, detected at the systemic velocity of $54 \mathrm{~km} \mathrm{~s}^{-1}$ is shown in Fig. 1 overlayed on a $\mathrm{H} \alpha$ image (cf. Goudis et al. 1983), This H I feature indicates the location of the ionization front, similar to the optical [NII] emission, and appears also to be related to molecular gas at the same velocity (SHWB). A second $\mathrm{H}$ I structure, observed at $63 \mathrm{~km} \mathrm{~s}^{-1}$, consists of clumps that surround a major part of the shell and the southern bar of NGC 2359. The dynamics of the nebula are consistent with the momentum conserving case or with an intermediate case between energy and momentum conservation.

Our results are in general agreement with the scenario for NGC 2359 described by Dufour (1989).

\section{References}

Dufour, R. 1989, RevMexAA 18, 87

Goudis, C., Hippelein, H., Münch, G. 1983, A\&A 117, 127

Goudis, C., Christopoulou, P.E., Meaburn, J., Dyson, J.E. 1994, A\&A 285, 631

Schneps, M.H., Haschick, A.D., Wright, E.L., Barrett, A.H. 1981, ApJ 243, 184 